\title{
On Fischer-type determinantal inequalities for accretive-dissipative matrices
}

Jianming $\mathrm{Xue}^{1^{*}}$ and Xingkai Hu${ }^{2}$

"Correspondence:

xuejianming104@163.com

'Oxbridge College, Kunming

University of Science and

Technology, Kunming, Yunnan 650106, P.R. China

Full list of author information is

available at the end of the article

\section{Abstract \\ This paper aims to give some refinements of recent results on Fischer-type determinantal inequalities for accretive-dissipative matrices.}

MSC: $15 \mathrm{~A} 45$

Keywords: accretive-dissipative matrix; Fischer determinantal inequality; Buckley matrix

\section{Introduction}

Let $M_{n}(C)$ be the set of $n \times n$ complex matrices. For any $A \in M_{n}(C)$, the conjugate transpose of $A$ is denoted by $A^{*}$. $A \in M_{n}(C)$ is accretive-dissipative if it has the Hermitian decomposition

$$
A=B+i C, \quad B=B^{*}, \quad C=C^{*},
$$

where both matrices $B$ and $C$ are positive definite. Conformally partition $A, B, C$ as

$$
\left(\begin{array}{ll}
A_{11} & A_{12} \\
A_{21} & A_{22}
\end{array}\right)=\left(\begin{array}{ll}
B_{11} & B_{12} \\
B_{12}^{*} & B_{22}
\end{array}\right)+i\left(\begin{array}{ll}
C_{11} & C_{12} \\
C_{12}^{*} & C_{22}
\end{array}\right),
$$

such that all diagonal blocks are square. Say $k$ and $l(k, l>0$ and $k+l=n)$ the order of $A_{11}$ and $A_{22}$, respectively, and let $m=\min \{k, l\}$. In this article, we always partition $A$ as in (1.2).

If $B=I_{n}$ in (1.1), then an accretive-dissipative matrix $A \in M_{n}(C)$ is called a Buckley matrix.

Let $A=\left(\begin{array}{ll}A_{11} & A_{12} \\ A_{21} & A_{22}\end{array}\right) \in M_{n}(C)$. If $A_{11}$ is invertible, then the Schur complement of $A_{11}$ in $A$ is denoted by $A / A_{11}:=A_{22}-A_{21} A_{11}^{-1} A_{12}$. For a nonsingular matrix $A$, its condition number is denoted by $k(A):=\sqrt{\frac{\lambda_{\max }\left(A^{*} A\right)}{\lambda_{\min }\left(A^{*} A\right)}}$, which is the ratio of the largest and the smallest singular value of $A$. For Hermitian matrices $B, C \in M_{n}(C)$, we write $B>(\geq) C$ to mean that $B-C$ is Hermitian positive (semi)definite.

If $A \in M_{n}(C)$ is positive definite, then the famous Fischer-type determinantal inequality ([1], p.478) states that

$$
\operatorname{det} A \leq \operatorname{det} A_{11} \cdot \operatorname{det} A_{22} \text {. }
$$

\section{(iㄹ) Springer}

(c) 2015 Xue and Hu. This article is distributed under the terms of the Creative Commons Attribution 4.0 International License (http://creativecommons.org/licenses/by/4.0/), which permits unrestricted use, distribution, and reproduction in any medium, provided you give appropriate credit to the original author(s) and the source, provide a link to the Creative Commons license, and indicate if changes were made. 
If $A \in M_{n}(C)$ is accretive-dissipative, Ikramov [2] first proved the determinantal inequality

$$
|\operatorname{det} A| \leq 3^{m}\left|\operatorname{det} A_{11}\right| \cdot\left|\operatorname{det} A_{22}\right| .
$$

If $A \in M_{n}(C)$ is accretive-dissipative, Lin [3] proved the determinantal inequality

$$
|\operatorname{det} A| \leq 2^{\frac{3 m}{2}}\left|\operatorname{det} A_{11}\right| \cdot\left|\operatorname{det} A_{22}\right|
$$

Recently, $\mathrm{Fu}$ and $\mathrm{He}$ ([4], Theorem 1) got a stronger result than (1.5) as follows.

Let $A \in M_{n}(C)$ be accretive-dissipative and partitioned as in (1.2). Then

$$
|\operatorname{det} A| \leq 2^{\frac{m}{2}}\left[1+\left(\frac{1-k}{1+k}\right)^{2}\right]^{m}\left|\operatorname{det} A_{11}\right| \cdot\left|\operatorname{det} A_{22}\right|
$$

where $k=\max (k(B), k(C))$.

For Buckley matrices, Ikramov [2] obtained the stronger bound

$$
|\operatorname{det} A| \leq\left(\frac{1+\sqrt{17}}{4}\right)^{m}\left|\operatorname{det} A_{11}\right| \cdot\left|\operatorname{det} A_{22}\right|
$$

In this paper, we will give refinements of (1.6) and (1.7) in Section 2. Other related studies of the Fischer-type determinantal inequalities for accretive-dissipative matrices can be found in [5-7].

\section{Main results}

We begin this section with the following lemmas.

Lemma 1 ([8], Property 6) Let $A \in M_{n}(C)$ be accretive-dissipative and partitioned as in (1.2). Then $A / A_{11}$ is also accretive-dissipative.

Lemma 2 ([2], Lemma 1) Let $A \in M_{n}(C)$ be accretive-dissipative as in (1.1). Then

$$
A^{-1}=E-i F, \quad E=\left(B+C B^{-1} C\right)^{-1}, \quad F=\left(C+B C^{-1} B\right)^{-1} .
$$

Lemma 3 ([9], Lemma 3.2) Let $B, C \in M_{n}(C)$ be Hermitian and assume $B$ is positive definite. Then

$$
B+C B^{-1} C \geq 2 C \text {. }
$$

Lemma $4([10],(6))$ Let $B=\left(\begin{array}{ll}B_{11} & B_{12} \\ B_{12}^{*} & B_{22}\end{array}\right)$ be Hermitian positive definite. Then

$$
B_{12}^{*} B_{11}^{-1} B_{12} \leq\left(\frac{1-k(B)}{1+k(B)}\right)^{2} B_{22}
$$

Lemma 5 ([3], Lemma 6) Let $B, C \in M_{n}(C)$ be positive semidefinite. Then

$$
|\operatorname{det}(B+i C)| \leq \operatorname{det}(B+C)
$$


Lemma 6 ([11], (1.2)) Let $a, b>0$. Then

$$
\left[1+\frac{(\ln a-\ln b)^{2}}{8}\right] \sqrt{a b} \leq \frac{a+b}{2} .
$$

Lemma 7 Let $B, C \in M_{n}(C)$ be positive definite. Then

$$
\operatorname{det}(B+C) \leq r^{n}|\operatorname{det}(B+i C)|
$$

where $r=\max _{1 \leq j \leq n}\left\{\sqrt{1+\frac{2}{2+\left(\ln \lambda_{j}\right)^{2}}}\right\}, \lambda_{j}$ are the eigenvalues of $B^{-1 / 2} C B^{-1 / 2}$, and $B^{1 / 2}$ means the unique positive definite square root of $B$.

Proof Letting $a=\lambda_{j}, b=\frac{1}{a}$ in Lemma 6 gives $1+\lambda_{j} \leq \sqrt{1+\frac{2}{2+\left(\ln \lambda_{j}\right)^{2}}}\left|1+i \lambda_{j}\right|, j=1, \ldots, n$. Then

$$
\begin{aligned}
\operatorname{det}(B+C) & =\operatorname{det} B \cdot \operatorname{det}\left(I+B^{-1 / 2} C B^{-1 / 2}\right) \\
& =\operatorname{det} B \cdot \prod_{j=1}^{n}\left(1+\lambda_{j}\right) \\
& \leq \operatorname{det} B \cdot \prod_{j=1}^{n}\left(\sqrt{1+\frac{2}{2+\left(\ln \lambda_{j}\right)^{2}}}\left|1+i \lambda_{j}\right|\right) \\
& \leq \operatorname{det} B \cdot \prod_{j=1}^{n}\left(r\left|1+i \lambda_{j}\right|\right) \\
& =r^{n} \operatorname{det} B \cdot\left|\operatorname{det}\left(I+i B^{-1 / 2} C B^{-1 / 2}\right)\right| \\
& =r^{n}|\operatorname{det}(B+i C)| .
\end{aligned}
$$

This completes the proof.

Theorem 1 Let $A \in M_{n}(C)$ be accretive-dissipative and partitioned as in (1.2). Then

$$
|\operatorname{det} A| \leq\left[1+\left(\frac{1-k}{1+k}\right)^{2}\right]^{m} r^{m}\left|\operatorname{det} A_{11}\right| \cdot\left|\operatorname{det} A_{22}\right|
$$

where $r=\max _{1 \leq j \leq n}\left\{\sqrt{1+\frac{2}{2+\left(\ln \lambda_{j}\right)^{2}}}\right\}, \lambda_{j}$ are the eigenvalues of $B^{-1 / 2} C B^{-1 / 2}, B^{1 / 2}$ means the unique positive definite square root of $B$, and $k=\max (k(B), k(C))$.

Proof By Lemma 2 and Lemma 3, we have

$$
\begin{aligned}
A / A_{11} & =A_{22}-A_{21} A_{11}^{-1} A_{12} \\
& =B_{22}+i C_{22}-\left(B_{12}^{*}+i C_{12}^{*}\right)\left(B_{11}+i C_{11}\right)^{-1}\left(B_{12}+i C_{12}\right) \\
& =B_{22}+i C_{22}-\left(B_{12}^{*}+i C_{12}^{*}\right)\left(E_{k}-i F_{k}\right)\left(B_{12}+i C_{12}\right)
\end{aligned}
$$

with

$$
E_{k}=\left(B_{11}+C_{11} B_{11}^{-1} C_{11}\right)^{-1} \leq \frac{1}{2} C_{11}^{-1}, \quad F_{k}=\left(C_{11}+B_{11} C_{11}^{-1} B_{11}\right)^{-1} \leq \frac{1}{2} B_{11}^{-1} .
$$


Set $A / A_{11}=R+i S$ with $R=R^{*}$ and $S=S^{*}$. By Lemma 1, we obtain

$$
\begin{aligned}
& R=B_{22}-B_{12}^{*} E_{k} B_{12}+C_{12}^{*} E_{k} C_{12}-B_{12}^{*} F_{k} C_{12}-C_{12}^{*} F_{k} B_{12}, \\
& S=C_{22}+B_{12}^{*} F_{k} B_{12}-C_{12}^{*} F_{k} C_{12}-C_{12}^{*} E_{k} B_{12}-B_{12}^{*} E_{k} C_{12} .
\end{aligned}
$$

It can be proved that

$$
\begin{aligned}
& \pm\left(B_{12}^{*} F_{k} C_{12}+C_{12}^{*} F_{k} B_{12}\right) \leq B_{12}^{*} F_{k} B_{12}+C_{12}^{*} F_{k} C_{12} \\
& \pm\left(C_{12}^{*} E_{k} B_{12}+B_{12}^{*} E_{k} C_{12}\right) \leq C_{12}^{*} E_{k} C_{12}+B_{12}^{*} E_{k} B_{12}
\end{aligned}
$$

Thus,

$$
R+S \leq B_{22}+2 B_{12}^{*} F_{k} B_{12}+C_{22}+2 C_{12}^{*} E_{k} C_{12} .
$$

As $B, C$ are positive definite, by Lemma 4 , we have

$$
B_{12}^{*} B_{11}^{-1} B_{12} \leq\left(\frac{1-k(B)}{1+k(B)}\right)^{2} B_{22}, \quad C_{12}^{*} C_{11}^{-1} C_{12} \leq\left(\frac{1-k(C)}{1+k(C)}\right)^{2} C_{22}
$$

Without loss of generality, we assume $m=l$, then

$$
\begin{aligned}
\left|\operatorname{det}\left(A / A_{11}\right)\right| & =|\operatorname{det}(R+i S)| \\
& \leq \operatorname{det}(R+S) \quad(\text { by Lemma } 5) \\
& \leq \operatorname{det}\left(B_{22}+2 B_{12}^{*} F_{k} B_{12}+C_{22}+2 C_{12}^{*} E_{k} C_{12}\right) \quad(\text { by }(2.3)) \\
& \leq \operatorname{det}\left(B_{22}+B_{12}^{*} B_{11}^{-1} B_{12}+C_{22}+C_{12}^{*} C_{11}^{-1} C_{12}\right) \quad(\text { by }(2.2)) \\
& \leq \operatorname{det}\left\{\left[1+\left(\frac{1-k(B)}{1+k(B)}\right)^{2}\right] B_{22}+\left[1+\left(\frac{1-k(C)}{1+k(C)}\right)^{2}\right] C_{22}\right\} \quad \text { (by (2.4)) } \\
& \leq\left[1+\left(\frac{1-k}{1+k}\right)^{2}\right]^{m} \operatorname{det}\left(B_{22}+C_{22}\right) \\
& \leq\left[1+\left(\frac{1-k}{1+k}\right)^{2}\right]^{m} r^{m}\left|\operatorname{det}\left(B_{22}+i C_{22}\right)\right| \quad(\text { by Lemma } 7) \\
& =\left[1+\left(\frac{1-k}{1+k}\right)^{2}\right]^{m} r^{m}\left|\operatorname{det} A_{22}\right|,
\end{aligned}
$$

where $k=\max (k(B), k(C))$.

The proof is completed by noting $|\operatorname{det} A|=\left|\operatorname{det} A_{11}\right| \cdot\left|\operatorname{det}\left(A / A_{11}\right)\right|$.

Remark 1 Because of $r \leq \sqrt{2}$, inequality (2.1) is a refinement of inequality (1.6).

Theorem 2 Let $A \in M_{n}(C)$ be accretive-dissipative and partitioned as in (1.2) with $B_{12}=0$.

Then

$$
|\operatorname{det} A| \leq\left(\frac{\sqrt{17}+1}{4}\right)^{m}\left|\operatorname{det} A_{11}\right| \cdot\left|\operatorname{det} A_{22}\right|
$$




\section{Proof Compute}

$$
\begin{aligned}
|\operatorname{det} A|= & |\operatorname{det}(B+i C)| \\
= & \operatorname{det} B \cdot\left|\operatorname{det}\left(I+i B^{-1 / 2} C B^{-1 / 2}\right)\right| \\
\leq & \left(\frac{\sqrt{17}+1}{4}\right)^{m} \operatorname{det} B \cdot\left|\operatorname{det}\left(I_{k}+i B_{11}^{-1 / 2} C_{11} B_{11}^{-1 / 2}\right)\right| \\
& \cdot\left|\operatorname{det}\left(I_{l}+i B_{22}^{-1 / 2} C_{22} B_{22}^{-1 / 2}\right)\right| \quad(\operatorname{by}(1.7)) \\
= & \left(\frac{\sqrt{17}+1}{4}\right)^{m}\left|\operatorname{det}\left(B_{11}+i C_{11}\right)\right| \cdot\left|\operatorname{det}\left(B_{22}+i C_{22}\right)\right| \\
= & \left(\frac{\sqrt{17}+1}{4}\right)^{m}\left|\operatorname{det} A_{11}\right| \cdot\left|\operatorname{det} A_{22}\right| .
\end{aligned}
$$

This completes the proof.

Remark 2 It is clear that inequality (2.5) is an extension of inequality (1.7).

\section{Competing interests}

The authors declare that they have no competing interests.

\section{Authors' contributions}

All authors contributed equally to the writing of this paper. All authors read and approved the final manuscript.

\section{Author details}

${ }^{1}$ Oxbridge College, Kunming University of Science and Technology, Kunming, Yunnan 650106, P.R. China. ${ }^{2}$ Faculty of Science, Kunming University of Science and Technology, Kunming, Yunnan 650500, P.R. China.

\section{Acknowledgements}

The authors wish to express their heartfelt thanks to the referees for their detailed and helpful suggestions for revising the manuscript. At the same time, we are grateful to Prof. Xiaorong Gan for her fruitful discussions.

Received: 23 March 2015 Accepted: 31 May 2015 Published online: 14 June 2015

\section{References}

1. Horn, RA, Johnson, CR: Matrix Analysis. Cambridge University Press, London (1985)

2. Ikramov, KD: Determinantal inequalities for accretive-dissipative matrices. J. Math. Sci. (N.Y.) 121, 2458-2464 (2004)

3. Lin, M: Fischer type determinantal inequalities for accretive-dissipative matrices. Linear Algebra Appl. 438, 2808-2812 (2013)

4. Fu, X, He, C: On some Fischer-type determinantal inequalities for accretive-dissipative matrices. J. Inequal. Appl. 2013, $316(2013)$

5. Lin, M: Reversed determinantal inequalities for accretive-dissipative matrices. Math. Inequal. Appl. 12, 955-958 (2012)

6. Drury, SW, Lin, M: Reversed Fischer determinantal inequalities. Linear Multilinear Algebra 62, 1069-1075 (2014)

7. Yang, J: Some determinantal inequalities for accretive-dissipative matrices. J. Inequal. Appl. 2013, 512 (2013)

8. George, A, Ikramov, KD: On the properties of accretive-dissipative matrices. Math. Notes 77, 767-776 (2005)

9. Zhan, X: Computing the extremal positive definite solutions of a matrix equation. SIAM J. Sci. Comput. 17, 1167-1174 (1996)

10. Zhang, F: Equivalence of the Wielandt inequality and the Kantorovich inequality. Linear Multilinear Algebra 48, 275-279 (2001)

11. Zou, L, Jiang, Y: Improved arithmetic-geometric mean inequality and its application. J. Math. Inequal. 9, 107-111 (2015) 